\title{
Unusual presentation of bilateral inferior shoulder dislocation following a trivial fall in an elderly female: a case report
}

Umesh Jayarajah, Chamikara Palkumbura, Arulprashanth Arulanantham, Aadil Faleel, Rukshan Sooriyarachchi

Department of Orthopaedics and Trauma, National Hospital of Sri Lanka, Colombo, Sri Lanka

Received: January 23, 2020

Accepted: March 16, 2020

\section{Correspondence}

Umesh Jayarajah

Department of Orthopaedics and Trauma, National Hospital of Sri Lanka, Colombo 10, Sri Lanka

Tel.: +94112691111

E-mail: umeshe.jaya@gmail.com

\section{Funding}

None

\section{Conflict of interest}

The Authors declare no conflict of interest

How to cite this article: Jayarajah $U, \mathrm{Pal}-$ kumbura C, Arulanantham A, et al. Unusual presentation of bilateral inferior shoulder dislocation following a trivial fall in an elderIy female: a case report. Journal of Gerontology and Geriatrics 2020;68:184-7. https://doi.org/10.36150/2499-6564-431

C Copyright by Società Italiana di Gerontologia e Geriatria (SIGG)

\section{(c) (1) (2) $\odot$}

\section{OPEN ACCESS}

This is an open access article distributed in accordance with the CC-BY-NC-ND (Creative Commons Attribution-NonCommercial-NoDerivatives 4.0 International) license. The article can be used by giving appropriate credit and mentioning the license, but only for non-commercial purposes and only in the original version. For further information: https://creativecommons.org/licenses/by-nc-nd/4.0/deed.en
Background. Bilateral inferior dislocation of the shoulder joint is infrequent. This usually results following significant impact from forceful hyperabduction or direct axial loading in the abducted position. We describe the case of bilateral inferior shoulder dislocation following a trivial fall on outstretched arms in an elderly female. A 75-year-old female who was on treatment for type 2 diabetes and hypertension, presented to the emergency department with painful inability to move both shoulders following a trivial fall. Her both shoulders were fixed at the 90 degree abducted position, the acromion processes were prominently palpable and both humeral heads were palpable in the axillae. $X$-ray confirmed the diagnosis of bilateral inferior dislocation of the shoulder joint. Closed reduction was successfully achieved using a single step levering manoeuvre with one hand applying traction at 90 degree abduction and the other hand levering the humeral head towards the glenoid. Post-procedure was uneventful with satisfactory functional outcomes. We described an unusual presentation of bilateral inferior dislocation of the shoulder joint following a trivial fall in an elderly female. The single step levering manoeuvre was successful in achieving reduction.

Key words: bilateral inferior shoulder dislocation, elderly, luxatio erecta, trivial fall, inferior dislocation, case report

\section{INTRODUCTION}

The shoulder joint is capable of the widest range of motion, and thus, dislocations are commonly seen in clinical practice. Anterior dislocation is the commonest form (95-97\%) followed by posterior dislocation (2-4\%) which is usually seen after seizures or electroconvulsive therapy ${ }^{1}$. The inferior dislocations known as luxatio erecta are infrequent constituting only $0.5 \%$ of all shoulder dislocations ${ }^{2}$. However, bilateral inferior dislocation of the shoulder joint is rare ${ }^{3}$. These usually result following significant impact from forceful hyperabduction or direct axial loading in the abducted position. We report an unusual occurrence of bilateral inferior shoulder dislocation following a trivial fall in an elderly female.

\section{CASE PRESENTATION}

A 75-year-old female presented to the emergency department with painful 
inability to move both shoulders. She slipped and fell forwards with outstretched arms while attempting to sit on a chair. She was on treatment for type 2 diabetes mellitus and hypertension. She had moderate pain and both shoulders were fixed at the 90 degree abducted position - "the hands up" position. Her acromion processes were prominently palpable and both humeral heads were palpable in the axillae. There was no neurovascular involvement. X-ray showed bilateral inferior dislocation of the shoulder joint with both humeral heads under the glenoids (Fig. 1). Based on the clinical findings and imaging, a diagnosis of uncomplicated bilateral shoulder dislocation was made.

Closed reduction was achieved under a short general anaesthesia by a single step levering manoeuvre in the operation room. We did not attempt reduction without sedation as patient was in pain. The humeral head was palpated in the axilla. One hand was placed medial to it while the other hand was placed at the proximal forearm close to the elbow joint. The humeral head was levered cephalic and outwards towards the glenoid while applying traction at 90 degree abduction using the other hand (Fig. 2).

Post-reduction X-ray confirmed successful relocation and there was no post-procedure complications (Fig. 3). The shoulder was immobilised in internal rotation for 3 weeks after reduction and the patient was started on physiotherapy. The patient had good family support to take care of her during the period of immobilisation. At 6 weeks she had satisfactory outcome with acceptable range of motion. She had only mild rotator cuff weakness therefore, magnetic resonance imaging was not performed. At 6 months follow up she had bilateral shoulder joint stiffness with limited range of movements of 100 degrees of abduction bilaterally. However, she was able to perform her daily activities without much restriction.

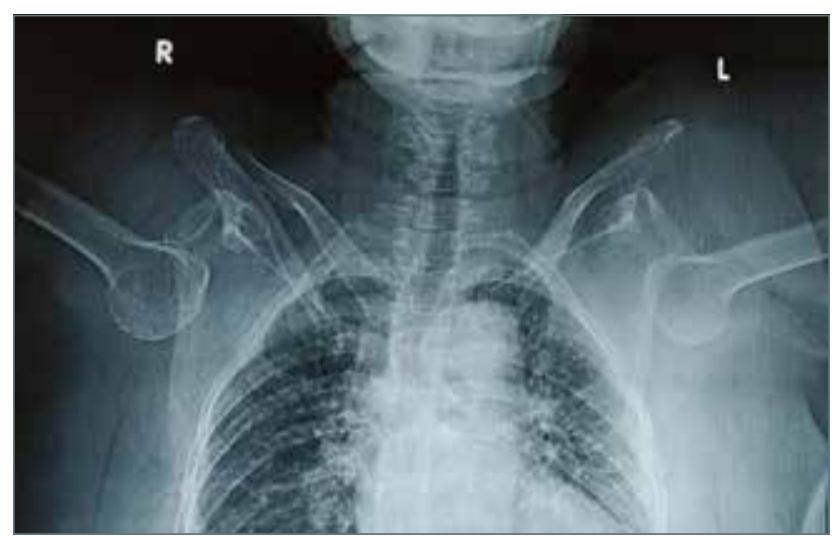

Figure 1. X-ray showing bilateral inferior dislocation of the shoulder joint with the humeral head under the glenoid.

\section{DISCUSSION}

Inferior shoulder dislocation, also known as luxatio erecta was first described in $1859^{2}$. It is rare and the available data are restricted to case reports and series ${ }^{2}$. We described the unusual presentation of bilateral inferior dislocation of the shoulder joint following a trivial fall in an elderly female. Post-reduction phase required good family support and furthermore, achieving acceptable range of movements in this elderly patient was challenging.

Two common types of mechanisms of injury have been documented ${ }^{2,4}$. In the direct mechanism, axial loading occurs on a fully abducted arm driving the humeral head through the weak joint capsule and inferior glenohumeral ligaments. This usually occurs following a fall from a height. It is frequently accompanied by rotator cuff tears and fractures of the greater tuberosity, acromion, coracoid, clavicle or inferior glenoid fossa ${ }^{3,5}$. In the indirect mechanism, an already abducted limb receives a strong abduction force which levers the proximal shaft of humerus over the acromion process driving the humeral head through the inferior capsule. This occurs after slipping from a moving vehicle while holding the gate bar ${ }^{3,6}$. Due to the direct forceful impact, the defect in the inferior capsule is rapid with an inline axis of entry, leading to a narrower defect in the capsule and the surrounding soft tissues. Entrapment of the humeral neck in the narrow defect may result in irreducibility ${ }^{3}$. In our patient, the pathomechanism of injury leading to bilateral inferior dislocation of the shoulder is unusual and unclear. The patient had a trivial fall forwards with the arms outstretched while trying to sit on a chair. Similar low energy impact resulting in unilateral and bilateral inferior dislocations have been described previously ${ }^{7,8}$. Both patients sustained a fall with the arms extended above the head and one patient had an associated greater tuberosity fracture ${ }^{7,8}$. The mechanism underlying the low energy impact causing inferior dislocations is unclear. However, this low energy mechanism may explain the absence of significant rotator cuff injury or neurovascular impairment in the above reported cases and in our patient ${ }^{7,8}$.

Several methods of closed reduction techniques have been described in literature. Commonest method described is axial traction on the abducted humerus and counter traction mobilising the torso ${ }^{2}$. Other method is a two-step manoeuvre which initially levers the humeral head anterior to the glenoid and subsequently follows the reduction techniques as for anterior dislocations ${ }^{9}$. However, there is no consensus for the optimal reduction technique for inferior shoulder dislocations. We used a single step levering manoeuvre with one hand applying traction at 90 degree abduction and the other hand levering the humeral head cephalic and outwards 


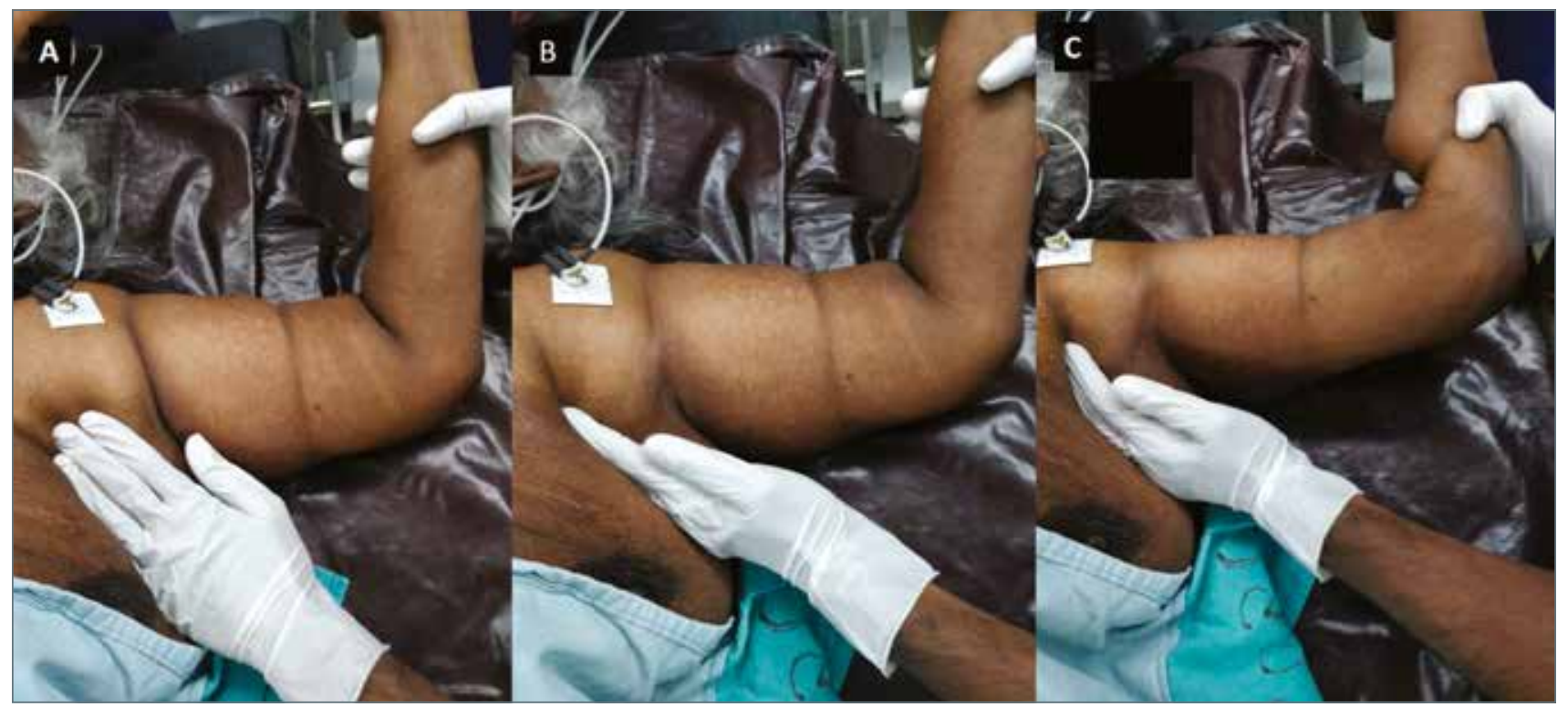

Figure 2. Closed reduction by a single step levering manoeuvre. A) Palpation of the humeral head in the axilla, B) Placement of one hand medial to the humeral head while the other hand holding the forearm, C) Levering the humeral head cephalic and outwards towards the glenoid while applying traction at 90 degree shoulder abduction.

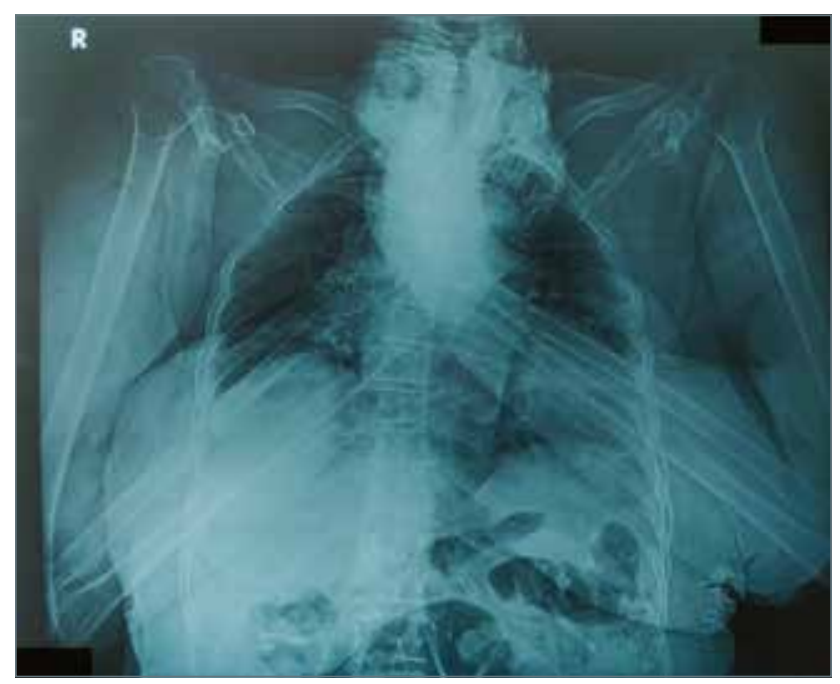

Figure 3. Post-reduction X-ray film.

towards the glenoid. This technique uses a levering force in addition to the traction force applied.

Neurovascular involvement is notorious following luxatio erecta probably due to the significant force involved. Although rare, axillary vessel injury may be associated requiring urgent vascular surgical intervention. The axillary nerve and brachial plexus injuries are the commonest neurological complications ${ }^{10}$. The treating surgeon should be aware of the associated bony and soft tissue injuries. Documentation of such injuries prior to reduction is essential for primary management of trauma and also for medico-legal purposes. Fortunately, our patient did not have any neurovascular involvement.

\section{CONCLUSIONS}

This case provided an unusual presentation of bilateral inferior shoulder dislocation in an elderly female. The pathomechanism of bilateral dislocation following a trivial injury is unusual. In this case, the single step levering manoeuvre was successful in achieving reduction. Ethics approval and consent to participate Not applicable

\section{CONSENT FOR PUBLICATION}

Informed written consent for publication was obtained from the patient prior to collecting information

Availability of data and material

All data generated or analysed during this study are included in this published article

\section{AUTHOR CONTRIBUTIONS}

Author UJ, CP, AA and AF contributed to collection of information and writing of the manuscript. Author RS contributed to writing and final approval of the manuscript. All authors have approved the final version before publication. 


\section{References}

1 Sherman SC, Schaider J. Shoulder dislocation and reduction. UpToDate, Waltham, MA 2010 (Accessed May 1, 2015).

2 Cift $H$, Soylemez S, Demiroglu $M$, et al. Rare inferior shoulder dislocation (Luxatio Erecta). Case Rep Orthop 2015;2015:624310. https://doi.org/ $10.1155 / 2015 / 624310$

3 Khedr H, Al-Zahrani A, Al-Zahrani A, et al. Bilateral irreducible inferior shoulder dislocation: a case report. Int $J$ Surg Case Rep 2017;31:124-7. https://doi.org/10.1016/j. ijscr.2017.01.025

4 Davids J, Talbott RD. Luxatio erecta humeri. A case report. Clin Orthop Relat Res 1990:144-9.

5 Yamamoto T, Yoshiya S, Kurosaka M, et al. Luxatio erecta (inferior dislocation of the shoulder): a report of 5 cases and a review of the literature. Am J Orthop (Belle Mead NJ) 2003;32:601-3.
6 Saxena V, Pradhan P. Bilateral luxatio erecta with greater tuberosity fracture: a case report. J Clin Orthop Trauma 2013;4:185-9. https://doi.org/10.1016/j.jcot.2013.10.003

7 Jalal Y, Zaimi S, Zine A, et al. Luxatio erecta with greater tuberosity fracture: a case report. Trauma Case Rep 2018;13:22-5. https://doi.org/10.1016/j.tcr.2017.11.002

8 Kessler A, Hinkley J, Houserman D,et al. Bilateral luxatio erecta humeri with acute anterior-inferior re-dislocation. Clin Pract Cases Emerg Med 2020;4:38-41. https://doi. org/10.5811/cpcem.2019.9.44205

9 Nho SJ, Dodson CC, Bardzik KF, et al. The two-step maneuver for closed reduction of inferior glenohumeral dislocation (luxatio erecta to anterior dislocation to reduction). J Orthop Trauma 2006;20:354-7. https://doi. org/10.1097/00005131-200605000-00010

10 Camarda L, Martorana U, D'Arienzo M. A case of bilateral luxatio erecta. J Orthopaed Traumatol 2009;10:97-9. https://doi.org/10.1007/s10195-008-0039-x 